Built-in surface electric field, piezoelectricity and photoelastic effect in GaN nanorods for nanophotonic devices

This article has been downloaded from IOPscience. Please scroll down to see the full text article.

2008 Nanotechnology 19235401

(http://iopscience.iop.org/0957-4484/19/23/235401)

The Table of Contents and more related content is available

Download details:

IP Address: 140.112.113.225

The article was downloaded on 26/06/2009 at 11:31

Please note that terms and conditions apply. 


\title{
Built-in surface electric field, piezoelectricity and photoelastic effect in GaN nanorods for nanophotonic devices
}

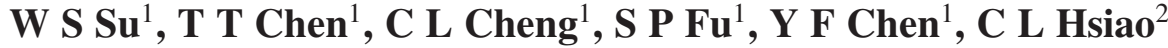 \\ and $\mathbf{L} \mathbf{W} \mathbf{T u}^{2}$ \\ ${ }^{1}$ Department of Physics, National Taiwan University, Taipei, Taiwan, Republic of China \\ 2 Department of Physics and Center for Nanoscience and Nanotechnology, \\ National Sun Yat-Sen University, Kaohsiung, Taiwan, Republic of China \\ E-mail: yfchen@phys.ntu.edu.tw
}

Received 29 November 2007, in final form 4 March 2008

Published 6 May 2008

Online at stacks.iop.org/Nano/19/235401

\begin{abstract}
Novel behaviors arising from the coupling between the built-in surface electric field, piezoelectricity, electron-hole pairs and external light beam were observed in GaN nanorods. An increase in the optical excitation density resulted in a blueshift in the photoluminescence spectra and a redshift in the frequency of the $\mathrm{GaN}_{1}(\mathrm{LO})$ phonon. The underlying mechanism was attributed to the screening of the built-in surface electric field by photoexcited carriers and, through the converse piezoelectric effect, a reduction in the internal strain. The existence of the built-in surface electric field in GaN nanorods was confirmed by scanning Kelvin probe microscopy. Our results firmly establish the existence of the photoelastic effect in GaN nanorods. In addition to underpinning the principle for applications in nanophotonic devices, this discovery also draws attention to the novel effects arising from the inherent large surface-to-volume ratio of nanostructures, which is possibly applicable to many other nanomaterials.
\end{abstract}

\section{Introduction}

Low-dimensional nanometer-scale materials have attracted intense interest in the past decade because of their enormous potential for applications in various electronic and optoelectronic nanodevices [1-5]. GaN and other related nitrides with direct bandgaps, large breakdown fields and high saturation velocities are the ideal candidates for efficient nanoscale ultraviolet/visible light emitters, hard radiation detectors and high-temperature nanoelectronic devices [6-8]. These devices were based primarily on zero-dimensional quantum dots and two-dimensional quantum well structures. Recently, the study of GaN has been extended to GaN-based nanorods and nanowires [9-11]. Practical applications of GaN nanorods include ultraviolet, blue and white light-emitting diodes (LEDs), laser diodes (LDs), nanosized vertical-cavity surface-emitting lasers (VCSEL) and many others [12-14]. However, before the potential applications of the nanorods can be fully realized, a better understanding of their unique properties is required. One of the outstanding characteristics which needs to be explored is their piezoelectricity, which has been studied in a rather limited fashion, especially for lowdimensional nitride materials. It is well known that $\mathrm{GaN}$ is a piezoelectric material in which an electric field can induce a strain, and vice versa [7, 15]. In general, spontaneous and piezoelectric polarizations exist in wurtize group III nitride epifilms, which can greatly modify the optical and electrical properties of those materials. Polarization is present along the hexagonal $c$ axis as a consequence of the noncentrosymmetry and the large ionicity of the covalent bonds. For the nitride film grown on sapphire or silicon, the lattice mismatch is quite large, and the stress due to the lattice mismatch relaxes at a film thickness of less than several nanometers. Even though the spontaneous polarization may play a significant role in thin quantum wells grown along the $c$ axis, it may become negligible in the case of nanorods grown along the $c$ axis. Because the nanorods inherently have a large side-wall surfaceto-volume ratio, the effect of the built-in electric field along the side-wall surface due to charges of surface defects now suppresses that of spontaneous polarization along the $c$ axis. 


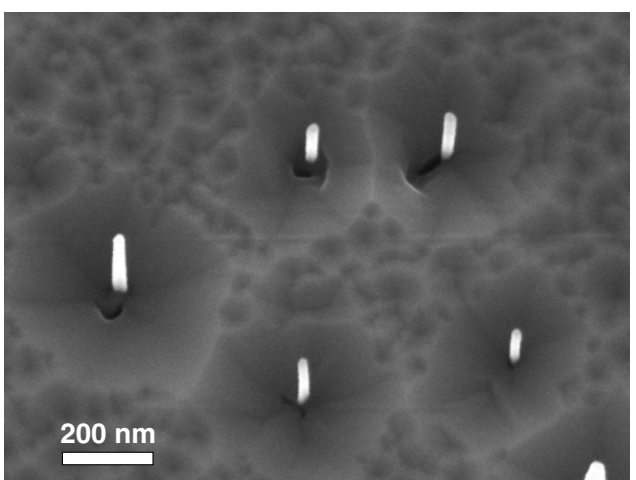

Figure 1. Scanning electron microscopy images of GaN nanorods.

Indeed, for $\mathrm{GaN}$ nanorods, a large built-in electric field on the side-wall surface has been demonstrated in several previous reports $[16,17]$.

In this paper, we present several intriguing behaviors arising from the unique intrinsic property of the coupling between the built-in electric field and the piezoelectric effect in $\mathrm{GaN}$ nanorods. Instead of applying an external electric field as is customary, we discover that the built-in electric field in the nanorods can be manipulated by changing the excitation power of a light beam. When the optical excitation density was increased, a redshift in the $A_{1}(L O)$ frequency and a blueshift in the micro-photoluminescence $(\mu$-PL) spectra of GaN nanorods were observed. These interesting results can be explained satisfactorily in terms of the screening of a built-in surface electric field, which results in a reduction of the biaxial strain in $\mathrm{GaN}$ nanorods through the converse piezoelectric effect. The variation of the biaxial strain can also be calculated by the Raman shift. The existence of the built-in surface electric field has been confirmed by scanning Kelvin probe microscopy (SKPM). Our results firmly establish the existence of the photoelastic effect in GaN nanorods, a fact which not only demonstrates one of the inherent properties of nanomaterials, but also underpins the background principle for their application in nanophotonic devices, including optical switches, optical modulators and solid state emitters.

\section{Experiment}

The samples studied here were grown on $\mathrm{Si}(111)$ wafers by plasma-assisted molecular beam epitaxy (MBE) (VeecoApplied Epi 930). The Ga source was 7N5 pure metal in a conventional effusion cell. $6 \mathrm{~N}$ pure $\mathrm{N}_{2}$ was further purified through a nitrogen purifier (Aeronex) and then fed into a plasma generator. The Si substrate was degreased and then lightly etched with diluted HF. A reconstructed $(7 \times 7)$ reflection high-energy electron diffraction pattern was obtained for the Si substrate after thermal treatment at $800^{\circ} \mathrm{C}$ to ensure excellent surface conditions. After low-temperature buffer layer deposition at $600^{\circ} \mathrm{C}$, high-temperature $\mathrm{GaN}$ growth started at $720^{\circ} \mathrm{C}$. Self-assembled vertical nanorods can be clearly seen in the field emission scanning electron microscope (FESEM) images as shown in figure 1. The average diameter of the studied nanorods was about $50 \mathrm{~nm}$ with a length

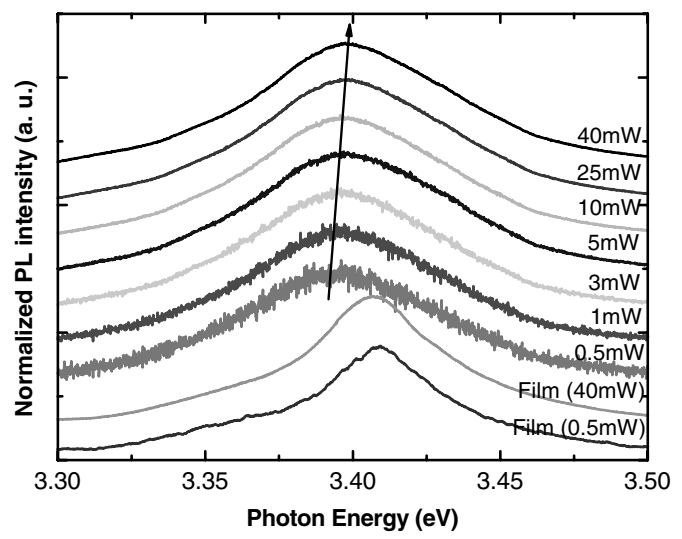

Figure 2. Excitation power dependence of photoluminescence spectra of GaN nanorods and epifilm.

of about $0.5 \mu \mathrm{m}$, and the average density of the nanorods was $\sim 10^{7} \mathrm{~cm}^{-2}$. A detailed distribution of the nanorods has been shown in our previous report [18]. Other detailed properties with various growth parameters can also be found elsewhere [10, 18, 19].

The morphology of GaN nanorods was determined by FESEM (JSM-6500F) equipped with an energy-dispersive spectrometer (EDS). The $\mu$-PL and $\mu$-Raman measurements were all performed at room temperature in a backscattering geometry by a Jobin Yvon T64000 system working in the triple-subtractive mode. The incident and scattered light propagated parallel to the $c$ axis, which was in turn normal to the growth surface. The sample was excited by a cw HeCd laser working at $325 \mathrm{~nm}$ and a neutral density filter was used to control the laser power. The surface potential was measured by SKPM using a standalone SMENA apparatus (NT-MDT) in non-contact mode. The silicon tips of the noncontact mode produced by NANOSENORS were coated with PtIr for measuring the Coulomb force. The thickness of the PtIr coating is about $23 \mathrm{~nm}$. The force constant is about $2.8 \mathrm{~N} \mathrm{~m}^{-1}$ and the resonance frequency is about $75 \mathrm{kHz}$.

\section{Results and discussion}

From our optical studies, several interesting behaviors were discovered in GaN nanorods. Figure 2 shows the powerdependent PL spectra of GaN nanorods at room temperature, and the spectrum of the GaN film is also shown for reference. At first glance, the linewidth of the epifilm is smaller than that of the nanorods, which implies the epifilm has a better crystalline quality than the nanorods. For the measurements of the GaN film, there were no nanorods in the region of the sample. To ensure the measurements of $\mathrm{GaN}$ nanorods, we have changed the focal plane height of the confocal microscope on the top of the GaN nanorods during the measurements. The excitation power was varied from 0.5 to $40 \mathrm{~mW}$ and the spot size of the laser beam was focused to about $40 \mu \mathrm{m}$ [2]. It is clear that the peak energy of the $\mathrm{GaN}$ nanorods was smaller than that of the GaN film by about $20 \mathrm{meV}$ at the same excitation power of $0.5 \mathrm{~mW}$. On increasing the excitation power, the PL peak energy of the GaN nanorods shows a 


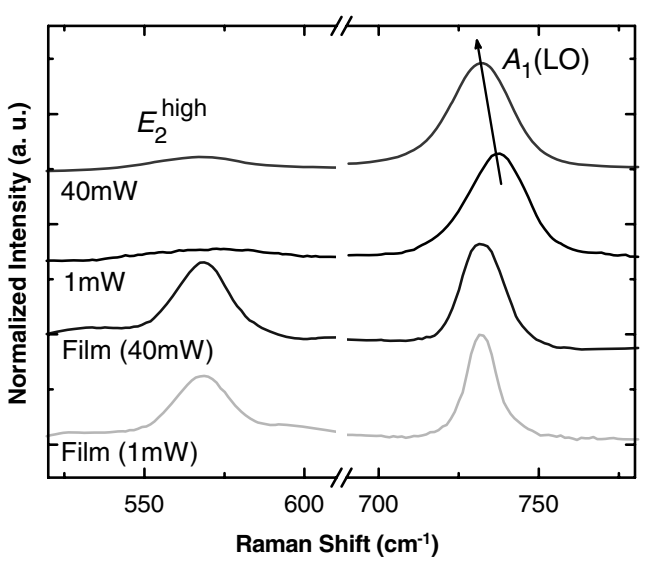

Figure 3. Room-temperature micro-Raman spectra of GaN nanorods under different excitation densities.

blueshift, while that of the GaN film was almost the same. This implication is important to explain our results as discussed below. Besides, the PL intensity also increases with increasing the excitation power, which can be easily understood due to an increase in the number of photoexcited electron-hole pairs.

In addition to the PL spectra, the Raman scattering spectra also show a very interesting behavior. According to group theory, $\mathrm{A}_{1}(z)+2 \mathrm{~B}_{1}+\mathrm{E}_{1}(x, y)+2 \mathrm{E}_{2}$ optical modes are predicted at the $\Gamma$ point of the Brillouin zone of hexagonal $\mathrm{GaN}$ [20]. Based on the Raman selection rules, only the $\mathrm{E}_{2}^{\text {low }}$, $\mathrm{E}_{2}^{\text {high }}$ and $\mathrm{A}_{1}(\mathrm{LO})$ modes can be observed with the $z(-,-) z$ scattering geometry in this experiment [21]. As shown in figure 3, the Raman signal near $733 \mathrm{~cm}^{-1}$ corresponds to the $\mathrm{A}_{1}$ (LO) mode and the broadened line located near $568 \mathrm{~cm}^{-1}$ corresponds to the $\mathrm{E}_{2}^{\text {high }}$ mode of $\mathrm{GaN}$. We can see that both of the $A_{1}(\mathrm{LO})$ and $\mathrm{E}_{2}^{\text {high }}$ modes of the GaN nanorods show a high-energy shift compared with those of the GaN film at the same excitation power of $1 \mathrm{~mW}$. Besides, it is found that the $\mathrm{A}_{1}(\mathrm{LO})$ and $\mathrm{E}_{2}^{\text {high }}$ phonon frequencies decrease with increasing excitation power and approach those of the GaN film, while those of the GaN film were almost the same at low and high excitation power. Additionally, it is clear that the linewidths of the $A_{1}(L O)$ and $E_{2}^{\text {high }}$ modes of the GaN film are smaller than those of the GaN nanorods, which again implies that the crystal quality of the GaN film is better than that of the GaN nanorods. This is consistent with the PL spectra as shown in figure 2.

Let us now examine the underlying mechanism responsible for the above observed behaviors. First of all, the effect of local heating due to absorption of the light beam can be ruled out because the corresponding PL peak exhibits a blueshift with increasing excitation power. If local heating is significant, the bandgap will decrease, which will result in a redshift of the PL spectra. Besides, the localized states arising from crystal imperfections can also be excluded. According to the previous reports in nitride semiconductors, all the blueshifts of PL peak energy with increasing temperature or excitation power were observed at temperatures below $100 \mathrm{~K}[22,23]$. However, this temperature is much lower than that of our measurement at room temperature. It is quite plausible that, in order to interpret the above observed behaviors, the inherent properties

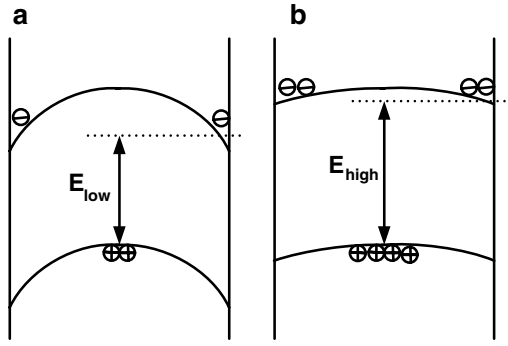

Figure 4. Effect of band bending on luminescent peak energy. (a) Low excitation power. (b) High excitation power.

of surface effects in nanorods must be taken into account. This is because the surface-to-volume ratio of the nanorod sample is much larger than that of the film. Therefore, we interpreted our results along this line of thought.

At first, another nanorod sample with a larger diameter of about $1 \mu \mathrm{m}$ was used to perform the EDS measurements, due to the limitation in the lateral resolution. We examined the $\mathrm{Ga}, \mathrm{N}$ and $\mathrm{O}$ compositions at the center and the edge on the top surface of the large rod. The result showed that there was about $4 \%$ of oxygen at the edge, while the composition of oxygen was zero in the center. It is worth noting that EDS measurement is not a surface-sensitive technique and the result shown here only serves as an indication that there exists a large amount of oxygen on the surface. According to previous calculations and experiments [24-26], the oxygen donor level is located at about $0.25 \mathrm{eV}$ above the conduction band minimum of $\mathrm{GaN}$. Therefore, as oxygen is adsorbed on the surface of nanorods, electrons will be injected into the nanorods. This process gives rise to positively charged oxygen ions at the surface, which leads to the formation of a built-in surface electric field and causes a downward band bending near the surface, as shown in figure 4(a). The downward band bending will accumulate and confine electrons close to the surface. The existence of built-in surface electric fields in GaN nanorods has also been demonstrated in several previous reports $[16,17]$.

In order to confirm the existence of the surface band bending, we have performed further SKPM measurements. For the SKPM measurement, we chose the same rod used for the EDS measurement due to the resolution limit. Figure 5(a) shows the surface potential distribution along the diagonal line at the top of the single nanorod as indicated in the inset. We can see that the surface potential near the side wall is higher than in the center. According to the energy band relation of metal-semiconductor contact, the surface potential $\varphi$ is equal to $\varphi=\phi_{\mathrm{m}}-\left(\chi+\Delta E_{\mathrm{fn}}\right)$, where $\chi$ is the electron affinity measured from the bottom of the conduction band to the vacuum level, $\phi_{\mathrm{m}}$ is the work function of the metal and $\Delta E_{\mathrm{fn}}$ is the Fermi level position relative to the bottom of the conduction band [27]. The electron affinity $\chi_{\mathrm{GaN}}$ of $\mathrm{GaN}$ is $4.2 \mathrm{eV}$ and the work function of PtIr is $5.5 \mathrm{eV}$ [28, 29]. Based on our measured surface potential, the $\Delta E_{\mathrm{fn}}$ can be calculated as shown in figure $5(\mathrm{~b})$, which indicates that the value of the $\Delta E_{\mathrm{fn}}$ difference between the side wall and center is about $25 \mathrm{meV}$. Therefore, the existence of the built-in surface electric field is established. 


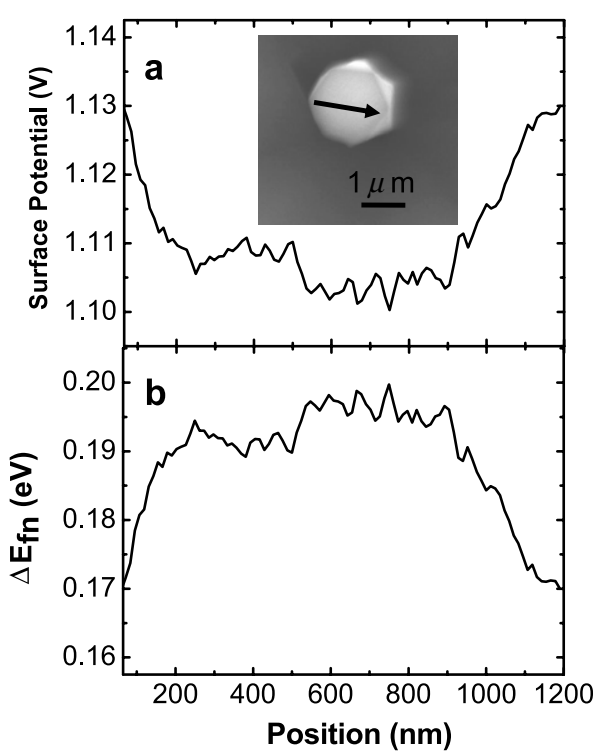

Figure 5. Scanning Kelvin probe microscope (SKPM) measurement on the top of the GaN nanorod. (a) The distribution of surface potential on the top of the nanorod. (b) The distribution of Fermi level position relative to the bottom of the conduction band on the top of the nanorod. The inset shows the top-view scanning electron microscope (SEM) image and scanning direction.

With the results obtained above, we now try to understand the blueshift of the PL peak with increasing excitation power. When the photoexcited electrons and holes are created, they will be spatially separated by the built-in electric field. Electrons will be confined at the surface of the nanorods, while the holes will be accumulated at the center. The spatially separated photoexcited carriers will result in a lower transition energy $\left(E_{\text {low }}\right)$ due to the band bending effect, which can be used to explain the experimental fact that the PL peak energy of the $\mathrm{GaN}$ nanorods is lower than that of the $\mathrm{GaN}$ film as observed in figure 2. Quite interestingly, the magnitude ( $20 \mathrm{meV}$ ) of the difference in the PL peak energies between the $\mathrm{GaN}$ nanorods and film is in good agreement with the value of $25 \mathrm{meV}$ for the surface band bending obtained from SKPM. This result further supports our proposed interpretation. In addition, due to the spatially separated electrons and holes, the built-in electric field will be screened, which then reduces the band bending as shown in figure 4(b). As a result, the emission energy increases with excitation power as observed in figure 2 . We can therefore see that the peculiar behaviors of the PL spectra in the $\mathrm{GaN}$ nanorods can be satisfactorily interpreted by the existence of a built-in surface electric field.

We are now ready to understand the interesting behaviors observed in the Raman scattering spectra as shown in figure 3. It is known that $\mathrm{GaN}$ is a piezoelectric material [7, 15]. With the converse piezoelectric effect, the built-in electric field will generate an internal compressive stress in $\mathrm{GaN}$ nanorods. The frequencies of phonon modes in $\mathrm{GaN}$ nanorods are thus blueshifted compared with those of $\mathrm{GaN}$ film as indicated in figure 3. In order to facilitate a more quantitative analysis, let us look at the relationship between the phonon frequency and applied strain. From Hooke's law, the magnitude of the frequency shift for each phonon mode is determined by the two

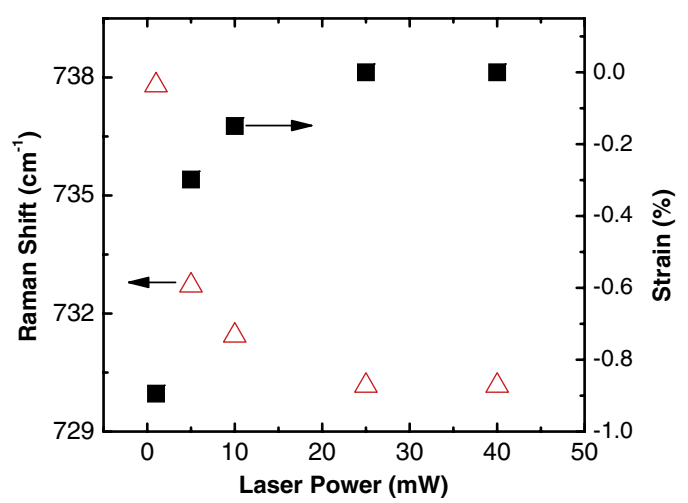

Figure 6. Relationship of $A_{1}(\mathrm{LO})$ phonon mode and internal strain versus excitation power.

(This figure is in colour only in the electronic version)

deformation potential constants $a$ and $b$ in the case of a given strain $\varepsilon_{x x}$ :

$$
\Delta \omega=2\left(a-b \frac{C_{13}}{C_{33}}\right) \varepsilon_{x x},
$$

where $\Delta \omega$ is the deviation in frequency of the $\mathrm{A}_{1}(\mathrm{LO})$ phonon, $C_{13}$ and $C_{33}$ are the elastic constants, $a$ is $-743 \mathrm{~cm}^{-1} \mathrm{GPa}^{-1}$, $b$ is $-735 \mathrm{~cm}^{-1} \mathrm{GPa}^{-1}$ and $2 C_{13} / C_{33}$ is 0.502 for $\mathrm{GaN}$ [30]. From the measurement of the $\mathrm{A}_{1}(\mathrm{LO})$ phonon at low excitation power, the measured $\Delta \omega$ is about $5 \mathrm{~cm}^{-1}$ and the corresponding strain $\varepsilon_{x x}$ is about $-0.9 \%$, as determined from equation (1). Therefore, we found that a sizable built-in strain was present in the GaN nanorods. The negative sign implies that a compressive biaxial strain existed in the GaN nanorods. According to the redshift of the $\mathrm{A}_{1}(\mathrm{LO})$ mode, the variation of the biaxial strain with increasing excitation density due to the screening of the built-in electric field can be calculated as shown in figure 6. Our results thus demonstrate that an external light beam can be used to manipulate the internal strain in the GaN nanorods, which proves the existence of the photoelastic effect in the GaN nanorods. Since the physics described here strongly underpin the underlying principles for optical modulators and the observed effects are strongly correlated with the inherent properties of nanoscale structures, our study thus opens up a new pathway for the application of nanomaterials.

\section{Conclusion}

In summary, we have clearly demonstrated that the coupling between the built-in electric field, piezoelectricity, electronhole pairs and external light beam in GaN nanorods can produce several intriguing behaviors. In particular, the existence of the photoelastic effect has been firmly established, which underpins the basic principle for the application of $\mathrm{GaN}$ nanorods in nanophotonic devices. Since the underlying mechanism arises from the existence of charged surface states, it is in all likelihood quite a common feature for most nanoscale materials. With the inherent large surface-to-volume ratio, the influence due to the variation of the surface electric field can be very significant, as demonstrated in our study. Our result 
can therefore be readily extended to the study of several other nanomaterials.

\section{Acknowledgment}

This work was supported by the National Science Council of the Republic of China.

\section{References}

[1] Edelstein A S and Cammarata R C 1996 Nanomaterials: Synthesis, Properties, and Applications (Philadelphia, PA: Institute of Physics)

[2] Morales A M and Lieber C M 1998 Science 279208

[3] Luryi S, Xu J and Zaslavsky A 2002 Future Trends in Microelectronics: The Nano Millennium (New York: Wiley-Interscience)

[4] Xia Y, Yang P, Sun Y, Wu Y, Mayers B, Gates B, Yin Y, Kim F and Yan H 2003 Adv. Mater. 15353

[5] Huang M H, Mao S, Feick H, Yan H, Wu Y, Kind H, Weber E, Russo R and Yang P 2001 Science 2921897

[6] Morkoc H and Mohammad S N 1995 Science 26751

[7] Strite S and Morkoc H 1992 J. Vac. Sci. Technol. B 101237

[8] Shur M S and Davis R F 2004 GaN-Based Materials and Device: Growth, Fabrication, Characterization and Performance (Singapore: World Scientific)

[9] Lin H M, Chen Y L, Yang J, Liu Y C, Yin K M, Kai J J, Chen F R, Chen L C, Chen Y F and Chen C C 2003 Nano Lett. 3537

[10] Tu L W, Hsiao C L, Chi T W, Lo I and Hsieh K Y 2003 Appl. Phys. Lett. 821601

[11] Park Y S, Park C M, Fu D J, Kang T W and Oh J E 2004 Appl. Phys. Lett. 855718
[12] Ristic J, Calleja E, Trampert A, Fernández-Garrido S, Rivera C, Jahn U and Loog K H 2005 Phys. Rev. Lett. 94146102

[13] Huang Z C, Chen J C, Mott D B and Shu P K 1996 Electron. Lett. 321324

[14] Nakamura S and Gasal G 1998 The Blue Laser Diode (Berlin: Springer)

[15] Pankove J I and Moustakas T D 1998 Semiconductors and Semimetals vol 50 (New York: Academic)

[16] Calarco R, Marso M, Richter T, Aykanat Ali I, Meijers R, Hart Andrè v d, Stoica T and Lüth H 2005 Nano Lett. 5981

[17] Cavallini A, Polenta L, Rossi M, Stoica T, Calarco R, Meijers R J, Richter T and Lüth H 2007 Nano Lett. 72166

[18] Hsiao C L, Tu L W, Chi T W, Seo H W, Chen Q Y and Chu W K 2006 J. Vac. Sci. Technol. B 24845

[19] Seo H W, Chen Q Y, Tu L W, Hsiao C L, Iliev M N and Chu W K 2005 Phys. Rev. B 71235314

[20] Hayes W and Laudon R 1978 Scattering of Light by Crystals (New York: Wiley)

[21] Wang D, Tin C-C, Williams J R, Park M, Park Y S, Park C M, Kang T W and Yang W C 2005 Appl. Phys. Lett. 87242105

[22] Eliseev P G, Perlin P, Lee J and Osinski M 1997 Appl. Phys. Lett. 71569

[23] Chen C H, Huang L Y, Chen Y F, Jiang H X and Lin J Y 2002 Appl. Phys. Lett. 801397

[24] Yang H C, Lin T Y and Chen Y F 2000 Phys. Rev. B 6212593

[25] McCluskey M D, Johnson N M, Van de Walle C G, Bour D P, Kneissl M and Walukiewicz W 1998 Phys. Rev. Lett. 804008

[26] Suski T, Perlin P, Skierbiszewski C, Wisniewski P, Dmowski L, Leszczynski M and Walukiewicz W 1999 Phys. Status Solidi b 216521

[27] Sze S Z 1969 Physics of Semiconductor Devices (New York: Wiley) p 368

[28] Liu Q Z and Lau S S 1998 Solid-State Electron. 42677

[29] Wildoer J W G, Harmans C J P M and van Kempen H 1997 Phys. Rev. B 5516013

[30] Wagner J-M and Bechstedt F 2000 Appl. Phys. Lett. 77346 